\title{
The duck hepatitis virus 5'-UTR possesses HCV- like IRES activity that is independent of elF4F complex and modulated by downstream coding sequences
}

\author{
Guangqing Liu ${ }^{1,2^{*}+}$, Emilio Yángüez ${ }^{3 \dagger}$, Zongyan Chen ${ }^{1}$ and Chuanfeng $\mathrm{Li}^{1}$
}

\begin{abstract}
Duck hepatitis virus (DHV-1) is a worldwide distributed picornavirus that causes acute and fatal disease in young ducklings. Recently, the complete genome of DHV-1 has been determined and comparative sequence analysis has shown that possesses the typical picornavirus organization but exhibits several unique features. For the first time, we provide evidence that the 626-nucleotide-long 5'-UTR of the DHV-1 genome contains an internal ribosome entry site (IRES) element that functions efficiently both in vitro and in mammalian cells. The prediction of the secondary structure of the DHV-1 IRES shows significant similarity to the hepatitis C virus (HCV) IRES. Moreover, similarly to HCV IRES, DHV-1 IRES can direct translation initiation in the absence of a functional elF4F complex. We also demonstrate that the activity of the DHV-1 IRES is modulated by a viral coding sequence located downstream of the DHV-1 5'-UTR, which enhances DHV-1 IRES activity both in vitro and in vivo. Furthermore, mutational analysis of the predicted pseudo-knot structures at the 3'-end of the putative DHV-1 IRES supported the presence of conserved domains II and III and, as it has been previously described for other picornaviruses, these structures are essential for keeping the normal internal initiation of translation of DHV-1.
\end{abstract}

\section{Background}

Duck hepatitis virus (DHV-1) is worldwide distributed and causes acute and fatal disease in young ducklings with severe economic impact in poultry industry. Although the disease was firstly reported in Long Island in 1949 [1], the complete genome of the causing pathogen was not determined until 2006 [2]. The DHV-1 genome is 7691-nucleotide-long and encodes a polyprotein of 2250 amino acids that is proteolitically processed to the individual viral proteins. Sequence analysis has assigned DHV-1 to a new genus in the Picornaviridae family [3]. Picornaviruses are a large family of viruses that include a number of important human and animal pathogens, such as Enterovirus, Rhinovirus, Cardiovirus, Aphthovirus, Hepatovirus, Parechovirus, Erbovirus, Kobuvirus and Teschovirus [4]. Although these viruses

\footnotetext{
* Correspondence: liugq@shvri.ac.cn

† Contributed equally

'Shanghai Veterinary Research Institute, Chinese Academy of Agricultural

Sciences, Shanghai, 200241, PR China

Full list of author information is available at the end of the article
}

have different host range and characteristics, they share common typical features, such us similar genome composition, genome structure and biological functions. The highly structured 5'-UTR of the picornavirus genome has been extensively characterized. The internal ribosome entry site (IRES) element located within this 5'-UTR directs internal initiation of viral protein synthesis in the infected cell [5].

The majority of host cell mRNAs are translated in a cap-dependent manner involving the recognition of their 5 '-cap structure by the eIF4F complex [6]. The eIF4F complex comprises three proteins: the eIF4E, the cellular cap-binding factor; the eIF4A, an RNA helicase responsible for the ATP-dependent elimination of secondary structures near the 5'-cap of the mRNAs; and the eIF4G, which functions as a scaffold to bind several factors such as the eIF3, the poly(A)-binding protein (PABP), the eIF4E or the eIF4A. Subsequently to the eIF4F binding to the 5'-cap structure, the $43 \mathrm{~S}$ pre-initiation complex is recruited to the mRNA, by its interaction with the eIF3,

\section{C) Biomed Central}


and the selected mRNAs are efficiently translated $[7,8]$. Initiation of translation is a major target for gene expression regulation [9-11] and viruses have evolved numerous unconventional mechanisms to preferentially recruit cellular translational machinery to the viral mRNA. Often, interactions of viral proteins with the components of the eIF4F complex and with the viral mRNA allow selective viral protein translation, blocking protein synthesis of the infected cell $[12,13]$. Translation initiation of picornaviruses proceeds by the direct interaction of the cellular machinery with the highly structured 5' IRES elements in the viral mRNAs. Structural insights coupled with biochemical studies have revealed that the IRES substitutes for the activities of some translation initiation factors. However, internal initiation strategies used by different members of this family differ in the requirement for eIF4F complex components. For instance, EMCV IRES recruits ribosomal machinery without the contribution of the cellular cap-binding protein eIF4E but requires active eIF2, eIF3, eIF4A and the central domain of eIF4G [14]. However, HCV IRES replaces the whole eIF4F complex and translation machinery is recruited by direct interaction of eIF3 and the viral mRNA [15-17].

According to their secondary structure, picornavirus IRES elements can be divided into four groups that display distinct biological properties $[18,19]$. The first group (class I) includes the IRES elements from enteroand rhinoviruses (e.g. poliovirus, PV) [20], while the second includes cardio- and aphthoviruses IRES elements (e.g. encephalomyocarditis virus, EMCV). The IRES element from hepatitis A virus (HAV) represents the third type of IRES [21,13], while the fourth group of picornavirus IRES elements has been recently described and includes porcine teschovirus-1 (PTV-1) Talfan strain, simian virus 2 [22-24], porcine enterovirus-8 (PEV-8) [25], simian picornavirus 9 and avian encephalomyelitis virus (AEV) [26]. It has been reported that the IRES elements of this group are similar to HCV IRES in sequence, function and predicted secondary structure.

Computer-assisted analysis revealed that the 626-ntlong 5'-UTR region of the DHV-1 RNA genome folds into a compact IRES-like structure with some similarities with PTV-1 and HCV-like IRESes [27]. The prediction of the RNA structure indicates that it contains the stem-loop structures found in other type II picornaviruses but not the clover leaf structures typically found in type I picornaviruses. These data suggest the presence of an IRES element in the 5'-UTR of DHV-1 RNA that could direct viral protein synthesis. To confirm this prediction, we have examined if the 5'-UTR region of DHV-1 genome could direct translation initiation both in in vivo and in vitro assays and we have characterized the presence of accessory regulatory sequences and the requirement for eIF4F complex components.

\section{Results}

Conserved secondary structure elements in DHV-1 and HCV-like IRES RNAs

Sequence analysis of the DHV-1 5'-UTR display a secondary structure with two major domains, II and III, which contain all the structural elements that have been described as crucial for internal translation initiation. The larger domain III consists on several branching high conserved hairpin stem-loops (III abcdef) (Figure 1A), which were also found in several members of the Picornaviridae family, such as the porcine teschovirus (PTV) and the avian encephalitis virus (AEV), and in some viruses from the Flaviviridae family, such as the classical swine fever virus (CSFV) and the hepatitis $C$ virus (HCV) [28,2,11,29].

\section{DHV-1 5'-UTR is able to initiate cap-independent translation}

In order to evaluate the IRES activity of the 5'-UTR region of the DHV-1 genome, a dicistronic reporter plasmid was constructed by the insertion of a cDNA corresponding to the DHV-1 5'-UTR (nts 1 to 626) between two reporter gene sequences, the first encoding CAT protein and the second encoding fLUC (DHV UTR). Dicistronic plasmid pGEM-CAT/EMCV/LUC (EMCV-IRES), which contains the EMCV IRES, was used as a positive control for internal translation initiation activity, and the plasmid pGEM-CAT/LUC (CAT/ LUC), which lacks any IRES sequence, was used as a negative control. The dicistronic plasmids were in vitro transcribed as indicated in Materials and Methods and the resultant RNAs were individually added to Flexi rabbit reticulocyte lysate (RRL) system (Promega). The in vitro translation of the reporter proteins was evaluated measuring ${ }^{35} \mathrm{~S}$-methionine incorporation. Capdependent translation from the first ORF was determined by the level of CAT expression and internal initiation activity of the different sequences included between the two reporter genes was estimated by the accumulation of fLUC. All the mRNAs expressed CAT efficiently as expected (Figure 2A). Moreover, plasmids containing the EMCV IRES and the DHV-1 5'-UTR sequence in sense orientation allowed the efficient expression of fLUC (Figure 2A). These results suggest that the DHV-1 5'-UTR sequence is able to efficiently initiate cap-independent protein synthesis in vitro.

To confirm and extend the results from these in vitro assays, the same dicistronic plasmids were tested in vivo by transient-expression experiments in mammalian cells (Figure 2B). The dicistronic plasmids were transfected into vFT7-infected BHK-21 cells, and $20 \mathrm{~h}$ post-transfection, cell extracts were prepared and protein synthesis was analyzed by SDS-PAGE and immunoblotting to detect CAT and LUC expression. As expected, all 


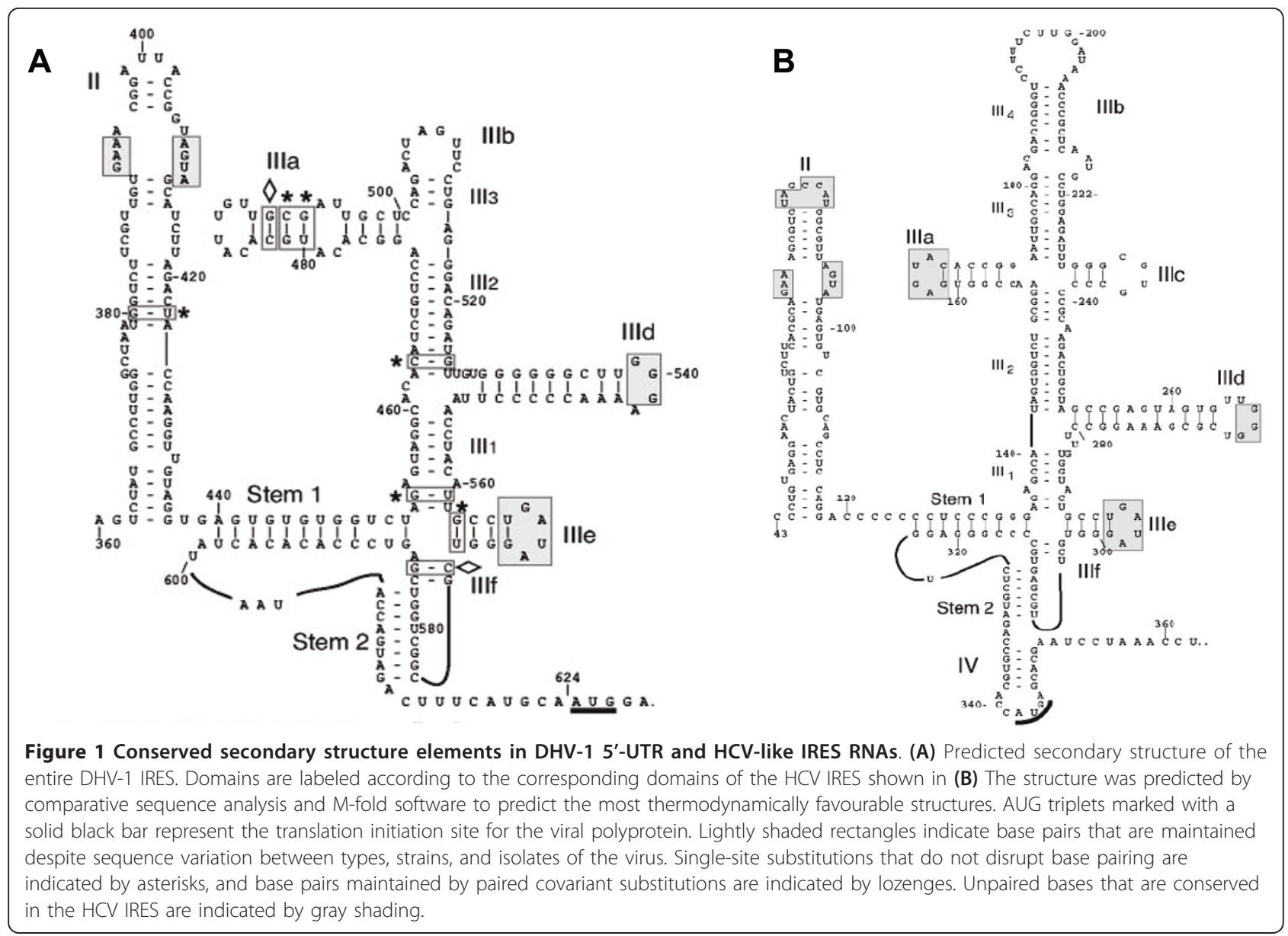

\section{A}

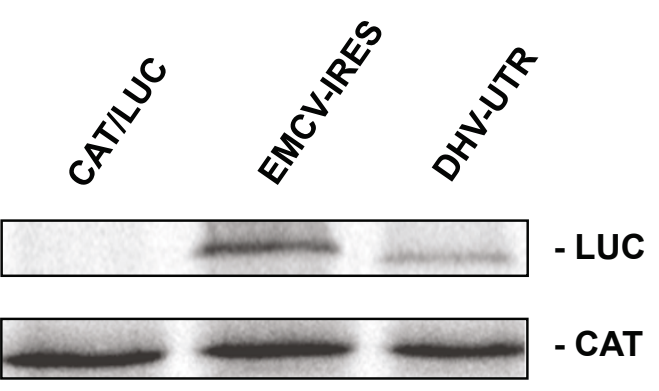

B
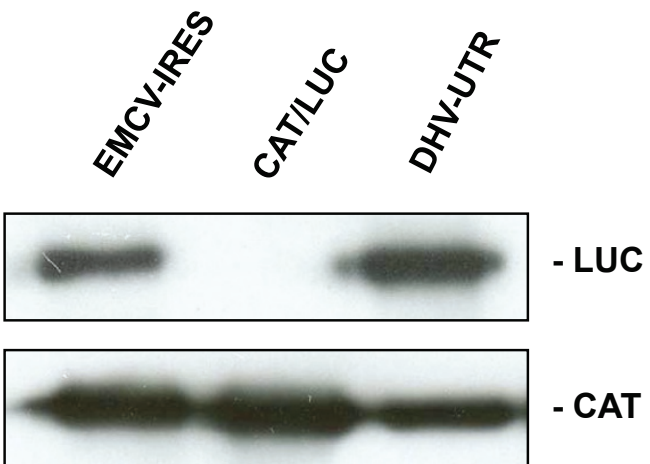

- CAT

Figure 2 The DHV 5'UTR initiates translation both in vitro and in vivo. (A) Dicistronic capped RNAs were in vitro generated from plasmid containing a CDNA insert corresponding to the DHV-1 5'-UTR (DHV UTR), PGEM-CAT/EMCV/LUC (EMCV-IRES) or pGEM-CAT/LUC (CAT/LUC). RNAS were individually added to Flexi rabbit reticulocyte lysate (RRL) system (Promega) and the in vitro translation of the reporter proteins was evaluated measuring ${ }^{35} \mathrm{~S}$-methionine incorporation. (B) The dicistronic plasmids were transfected into vFT7-infected BHK-21 cells, and $24 \mathrm{~h}$ posttransfection, cell extracts were prepared and protein synthesis was analyzed by SDS-PAGE and immunoblotting to detect CAT and fLUC expression. 
plasmids expressed CAT efficiently. Moreover, the DHV-1 5'-UTR and the EMCV IRES containing plasmids also produced efficient fLUC accumulation. These results indicated that the putative DHV-1 IRES element is able to efficiently initiate protein synthesis in vivo.

\section{Stem1, Stem2 and domain Ille are essential to the function of DHV-1 IRES}

Although there is only limited primary sequence relatedness among the 5'-UTRs of DHV-1, AEV and HCV, the structure of domain IIIe appears remarkably conserved among these viral IRESes $[2,35,11]$. It has been previously reported that domain IIIe is critical for the HCV, PTV-1 or AEV IRES-mediated initiation of translation. To evaluate the relevance of the putative IIIe region in DHV-1 IRES activity, the most important loop sequence within the IIIe region (GAUA) was mutated to AAAA (DHV IIIe mut) (Figure 3A) and the effect of the mutation on the translation initiation was evaluated. Mutant constructs were analyzed both in Flexi RRL
(Figure 3) and in DF-1 cells (Figure 4). The results reveal that these mutations partially inhibited translation initiation both in vitro and in vivo, confirming the relevance of domain IIIe for DHV-1 IRES activity. Surprisingly, the reduction in the translational activity of the DHV-1 IRES upon disruption of the domain IIIe (50\%) is moderate when compared with similar mutations in other type IV IRESes (90-95\%).

Different experiments carried out with other picornaviruses, such as the HCV, PTV or AEV, showed that stem 1 and stem 2 within the IRES structure are crucial for translation initiation. The disruption of the base pair interactions in these regions seriously damages the ability to initiate translation. To analyze the role of the putative stems loops of the DHV-1 IRES, different mutations were introduced to disrupt the predicted base pairing of stem 1 (DHV stem1 mut) and stem 2 (DHV stem 2 mut) (Figure 3A). The mutant constructs were analyzed by triplicate both in Flexi RRL (Figure 3) and in DF-1 cells (Figure 4). The results showed that both

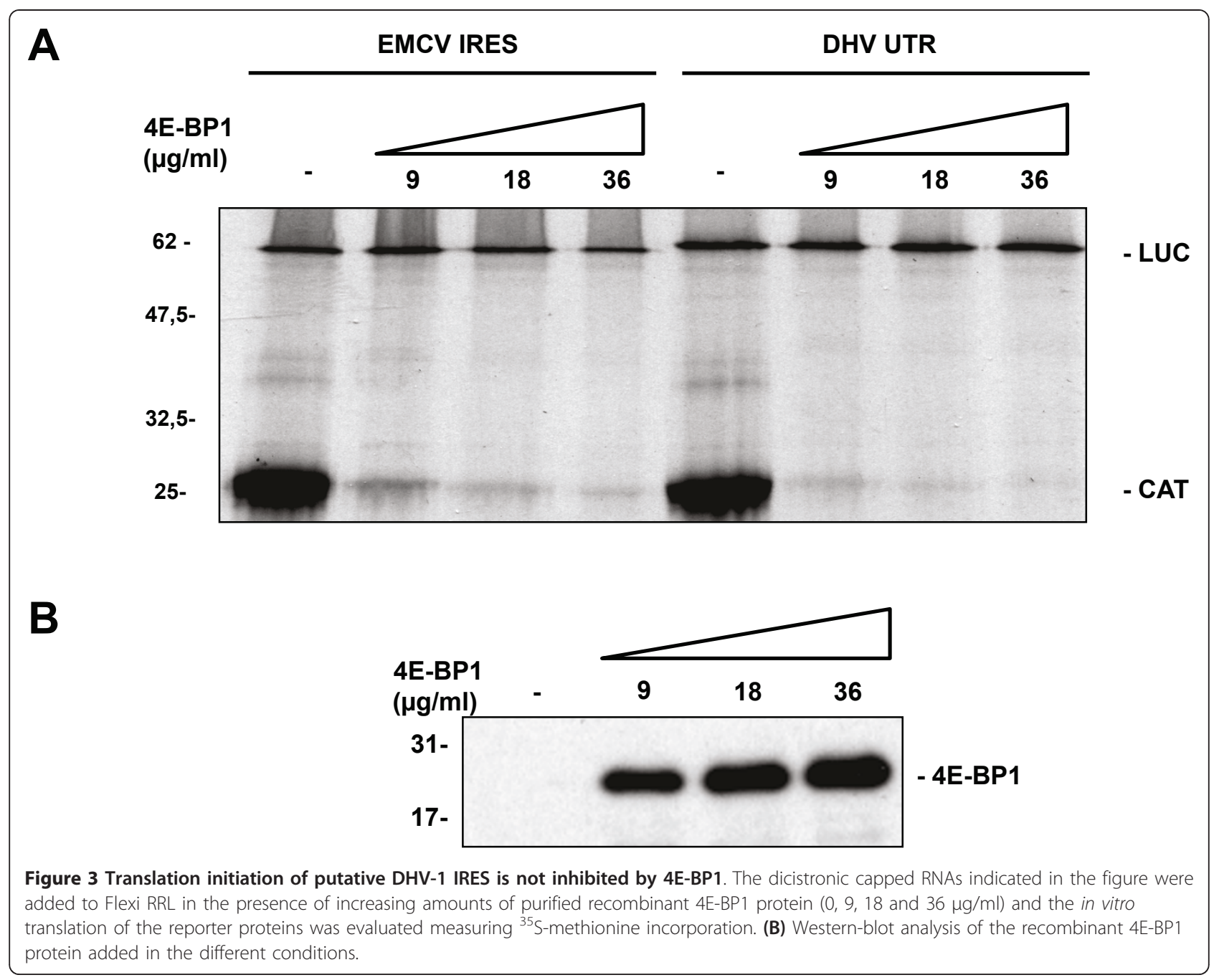




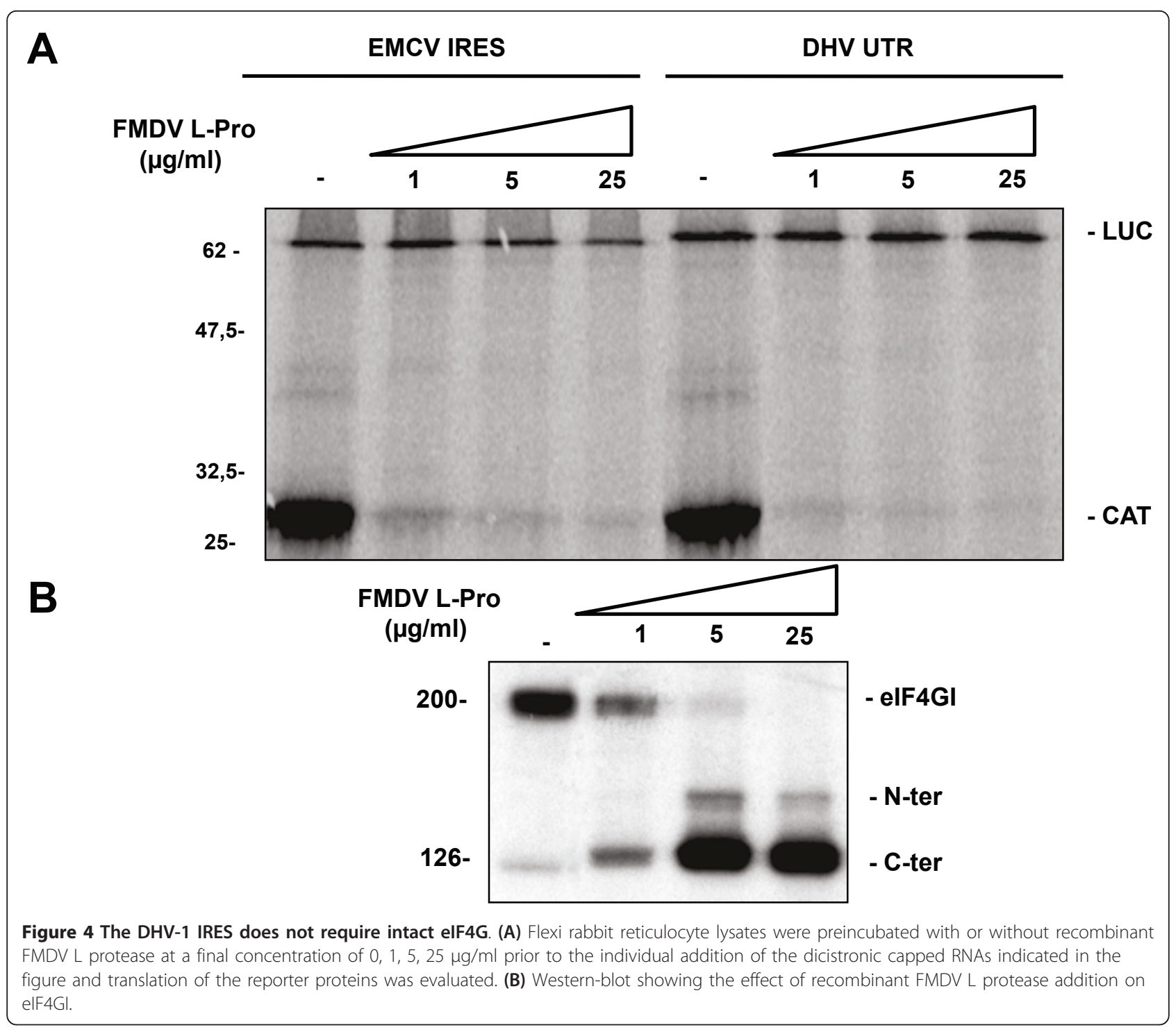

mutations resulted in a slight reduction in translation initiation ability of DHV-1 IRES both in vitro and in vivo. Moreover, the function of the IRES was partially recovered with the corresponding mutations that restore the structure (DHV stem 1 mut' and DHV stem 2 mut' respectively), confirming that the predicted pseudo-knot structures are formed in vivo and that they play a role in viral translation regulation. Once more, the reduction in the translational activity of the DHV-1 IRES upon disruption of these structures $(50 \%)$ is moderate when compared with similar mutations in other type IV IRESes (90-95\%).

\section{DHV-1 IRES activity is modulated by downstream coding} sequences

To further characterize the presence of accessory regulatory sequences downstream of the 5'-UTR of the viral genome, 4 additional dicistronic reporter plasmids were constructed, containing DHV-1 5'-UTR and 10, 40 or 60 nucleotides of the coding region adjacent to the viral 5'-UTR (DHV-1 UTR+10nt, UTR+40nt, UTR+60nt, respectively) (Figure 5). Initiating AUG codon of the DHV-1 IRES was mutated to allow the expression of the luciferase from its own AUG. The dicistronic plasmids were in vitro transcribed and individually added to Flexi rabbit reticulocyte lysate (RRL) system (Promega). The translation of the reporter proteins was evaluated measuring ${ }^{35} \mathrm{~S}$-methionine incorporation. Cap-dependent translation from the first ORF was determined by the level of CAT expression and internal initiation activity of the different sequences included between the two reporter genes was estimated by the accumulation of fLUC. All the constructs expressed CAT efficiently as expected but the efficiency of the DHV-1 UTR+10nt, 


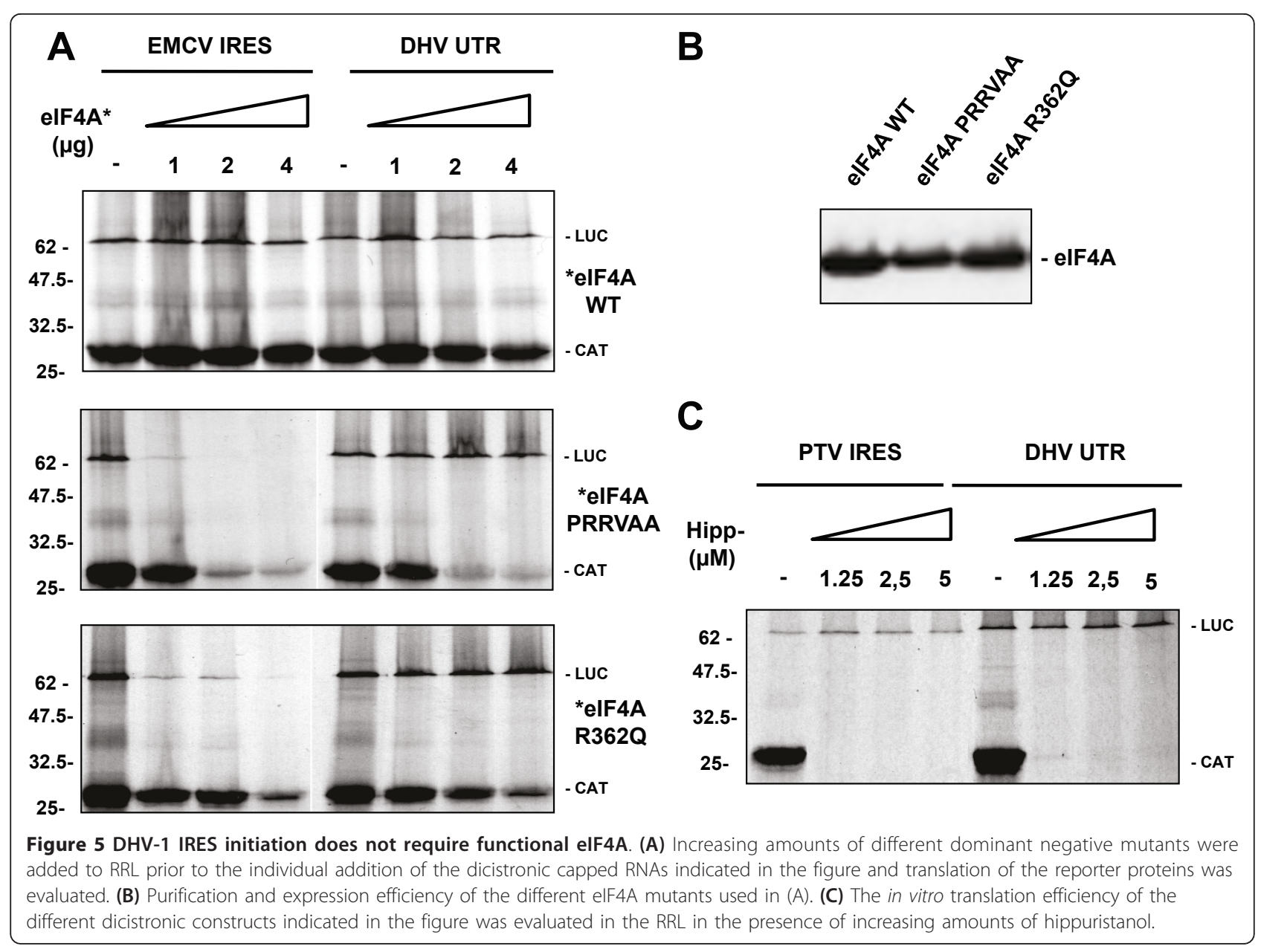

UTR+40nt and UTR+60nt sequences was stronger than that of the DHV-1 UTR (Figure 5A). Moreover, the dicistronic plasmids were transfected into vFT7-infected BHK-21 cells and, in order to evaluate the in vivo internal initiation activity, the accumulation of fLUC protein expressed from the different constructs was determined and corrected by CAT expression levels. The experiments were performed by triplicate. As observed in the in vitro experiments, the translation initiation efficiency of DHV-1 UTR+10nt, UTR+40nt and UTR+60nt sequences was stronger than that of the DHV-1 UTR (Figure 5B). These results indicate the presence of accessory regulatory sequences downstream the DHV-1 putative IRES, which are not required for translation initiation but positively modulate the IRES activity.

\section{Characterization of the putative DHV-1 IRES requirement} for elF4F complex components

Translation initiation of DHV-1 IRES is not inhibited by 4E-BP1

Picornavirus IRES-mediated translation is independent of the cellular cap-binding protein eIF4E. In order to evaluate the putative DHV-1 IRES requirement for the eIF4E, the in vitro translation efficiency of the dicistronic constructs was evaluated in RRL as described upon addition of increasing amounts of purified recombinant 4E-BP1 protein. The eIF4E-binding proteins (4E-BPs) are a family of three small polypeptides that inhibit cap-dependent translation by binding to the eIF4E, obstructing its interaction with eIF4G [30]. However, the internal translation initiation on IRES elements is not affected in these conditions. As shown in Figure 6A, whereas the recombinant 4E-BP1 efficiently inhibited the cap-dependent CAT expression in RRL, the EMCV IRES-directed and DHV-1 5'-UTR-directed translation were unaffected.

\section{The DHV-1 IRES does not require intact elF4G}

According to the prediction of the 5'-UTR secondary structure, DHV-1 could be included among the type IV picornaviruses. Previous studies have shown that the type IV IRES elements (HCV-like IRESes) do not require the eIF4G activity to direct translation initiation and, consequently, that are insensitive to the eIF4G cleavage induced by different viral proteases, such as the 


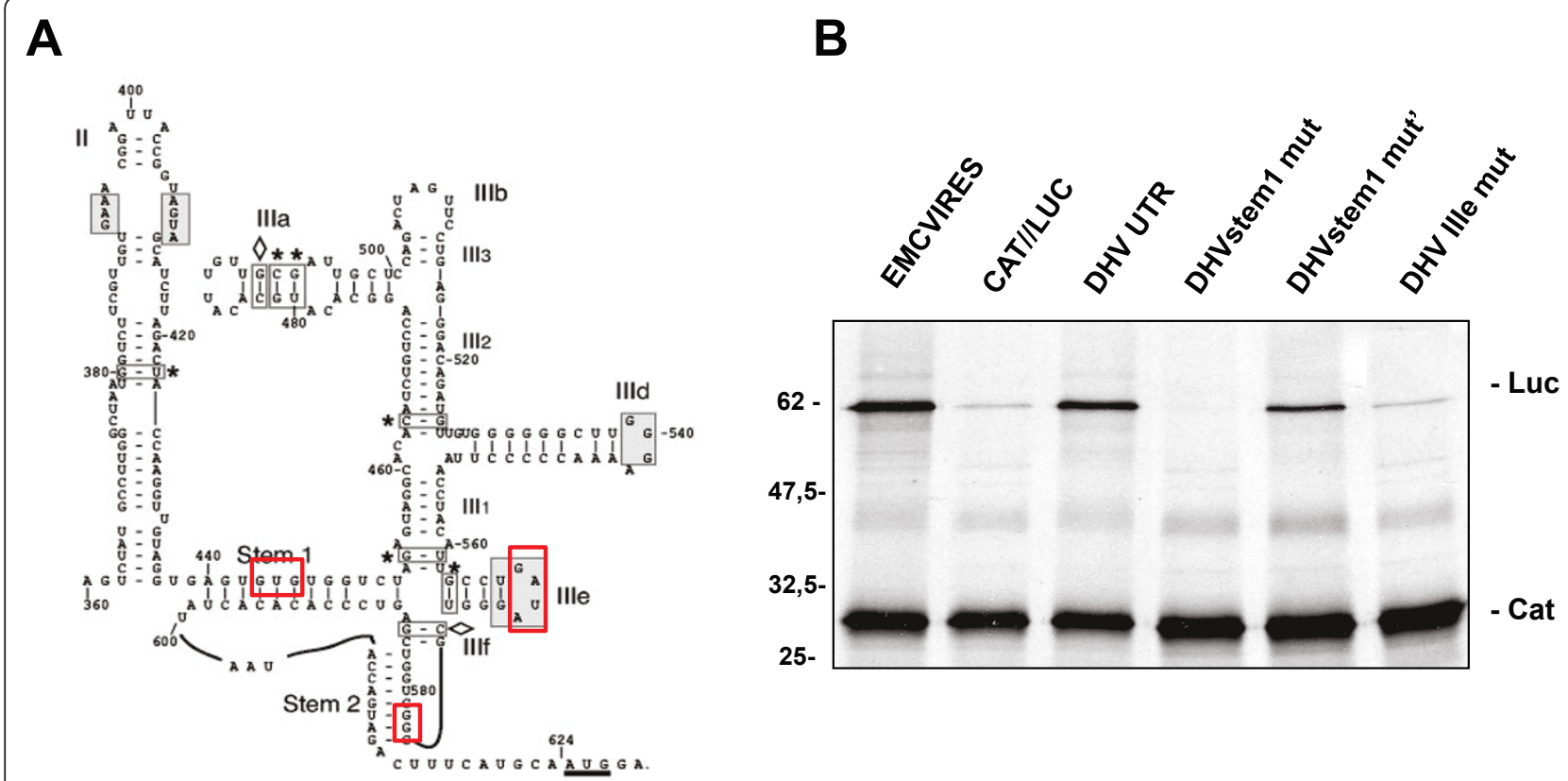

Figure 6 Predicted domain Ille and stem 1 on DHV-1 5'-UTR are required for in vitro IRES activity. (A) The position of the different mutations evaluated in Figures 7 and 8 is shown in red. (B) To evaluate the relevance of the predicted structures in DHV-IRES, a dicistronic plasmid, in which the most important loop sequence within the putative Ille region (GAUA) is mutated to AAAA (DHV Ille mut), was constructed. A different dicistronic plasmid containing mutations to disrupt the predicted base pairing of stem 1 (DHV stem 1 mut) and the corresponding reverse mutant that restores the structure (DHV stem1 mut') were also constructed. Mutant constructs were in vitro transcribed analyzed in RRL and translation of the reporter proteins was evaluated.

foot-and-mouth disease virus (FMDV) L protease. The FMDV L protease efficiently cleaves the eukaryotic eIF4G resulting in a partial inhibition of the cap-dependent translation while the IRES-directed translation is not inhibited in these conditions and it can even be enhanced [31]. To confirm that the translation of DHV$15^{\prime}$-UTR is cap-independent and to examine its eIF4G dependence, the effect of foot-and-mouth disease virus (FMDV) L protease addition in the RRL system was evaluated. The dicistronic RNA constructs indicated in Figure 7A were individually added to FMDV-L treated or control Flexi RRL and the translation of the reporter proteins was evaluated. Whereas the cap-dependent CAT expression was efficiently inhibited, the EMCVdirected and the DHV-1 5'-UTR-directed translation were insensitive to FMDV L protease addition. Western blot analysis was performed to control the efficiency of the eIF4G cleavage by FMDV L protease (Figure 7B). These results indicate that the DHV IRES directs capindependent internal initiation of translation and not requires an intact full-length eIF4G.

\section{DHV-1 IRES initiation does not require functional elF4A}

In order to evaluate the contribution of the remaining component of the eIF4F complex, the RNA helicase eIF4A, two different experimental in vitro approaches were used. Firstly, the effect on DHV-1 translation initiation of two different dominant negative mutants of eIF4A was evaluated. The dominant negative mutant PRRVAA contains a mutation in the conserved Ia region (PTRELA to PRRVAA) and is inactive in the ATP hydrolysis and the RNA unwinding activities [31]. The dominant negative mutant $\mathrm{R} 362 \mathrm{Q}$ contains a mutation in the conserved arginine in position 362 (R362Q) of the C-terminal region HRIGRXXR, and exhibits drastically reduced RNA binding and RNA helicase activity [32]. The dicistronic RNAs indicated in Figure 8A were individually added to control Flexi RRL or eIF4A dominant negative mutant-containing RRL and translation of the reporter proteins was evaluated. The EMCV IRES, a class 2 IRES dependent on eIF4A activity, was used as negative control. Whereas the cap-dependent CAT expression and the EMCV-directed translation were efficiently inhibited, DHV-1 the IRES-directed translation was insensitive to eIF4A dominant negative mutant.

To further analyze the eIF4A requirement and confirm these results, in vitro experiments were carried out using increasing amounts of a small molecule inhibitor of eIF4A. The hippuristanol is a sterol isolated from the coral Isis hippuris and identified via a high throughput screening for general translation inhibitors. It has been shown to block the eIF4A-dependent translation by inhibiting its RNA binding, ATPase, and helicase 


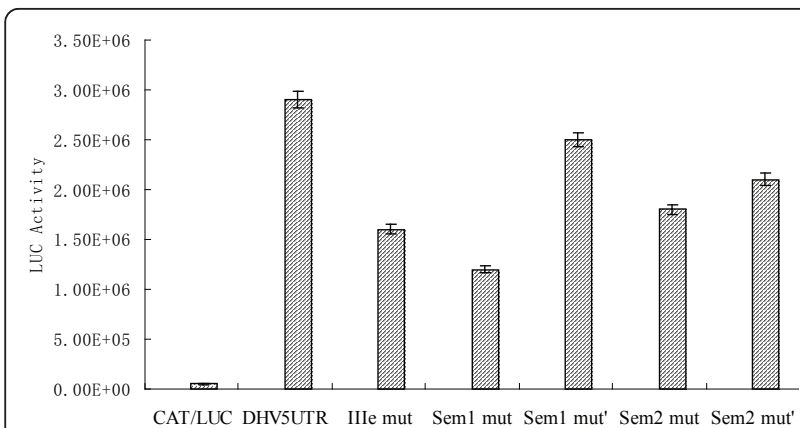

Figure 7 Predicted domain Ille, stem 1 and stem 2 on DHV-1 5'UTR are required for in vivo IRES activity. A dicistronic plasmid containing mutations to disrupt the predicted base pairing of stem 2 (DHV stem2 mut) and the corresponding reverse mutant that restores the structure (DHV stem2 mut') were constructed. In vivo translation efficiency of these new plasmids was evaluated, together with the plasmids depicted in Figure 7. Briefly, dicistronic plasmids were transfected into DF-1 cells and 48 hpt the accumulation of fLUC and CAT protein expressed from the different constructs was evaluated. fLUC expression was normalized by CAT expression levels.

activities by interaction with the C-terminal domain of the eIF4A [33,34]. The in vitro translation efficiency of the different dicistronic RNAs was evaluated in the RRL in the presence and in the absence of hippuristanol. In this experiment, PTV IRES, a class IV IRES which direct translation initiation independently of eIF4A, was used as positive control. Cap-dependent translation was efficiently inhibited by the eIF4A inhibitor, while PTV and DHV-1 IRES-directed translation were unaffected in these conditions (Figure 8C). These results indicate that,
DHV-1 5'-UTR, as well as the class IV HCV-like IRESes, do not require functional eIF4A to initiate translation.

\section{Discussion}

Picornavirus mRNAs are translated by an internal initiation mechanism, in which the ribosome enters directly at an internal site within the mRNA rather than scanning from the physical $5^{\prime}$ end. So far, internal ribosome entry sites (IRESs) have been identified for all the picornaviruses as well as an increasing number of cellular mRNAs. The IRES elements in picornaviruses have been located within the 5 ' non-coding region, where the sequence and the RNA secondary structure are well conserved among viral serotypes in each genera. Based on sequence alignments, the genome of DHV-1 showed a typical picornavirus genetic organization and a putative class IV IRES element within DHV-1 5'-UTR. Therefore, DHV-1 should use similar picornavirus-like strategies for the initiation of translation. In this paper, we demonstrate, for the first time, that the DHV-1 5'-UTR has typical IRES activity, and that it can drive the translation of a downstream reporter gene both in vitro and in vivo. According to the prediction of the IRES structure, the DHV-1 IRES belongs to the type IV HCV-like IRESes, but it shares some common features with the IRES of viruses included in different groups.

Some picornaviruses, such as the AEV, contain HCVlike IRES elements organized in two major secondary structure domains, II and III $[35,36,8]$, in which all the structural elements crucial for initiation of translation are located. Mutations within the putative loop of domain IIIe and stem1, 2 inhibited the DHV-1 IRES

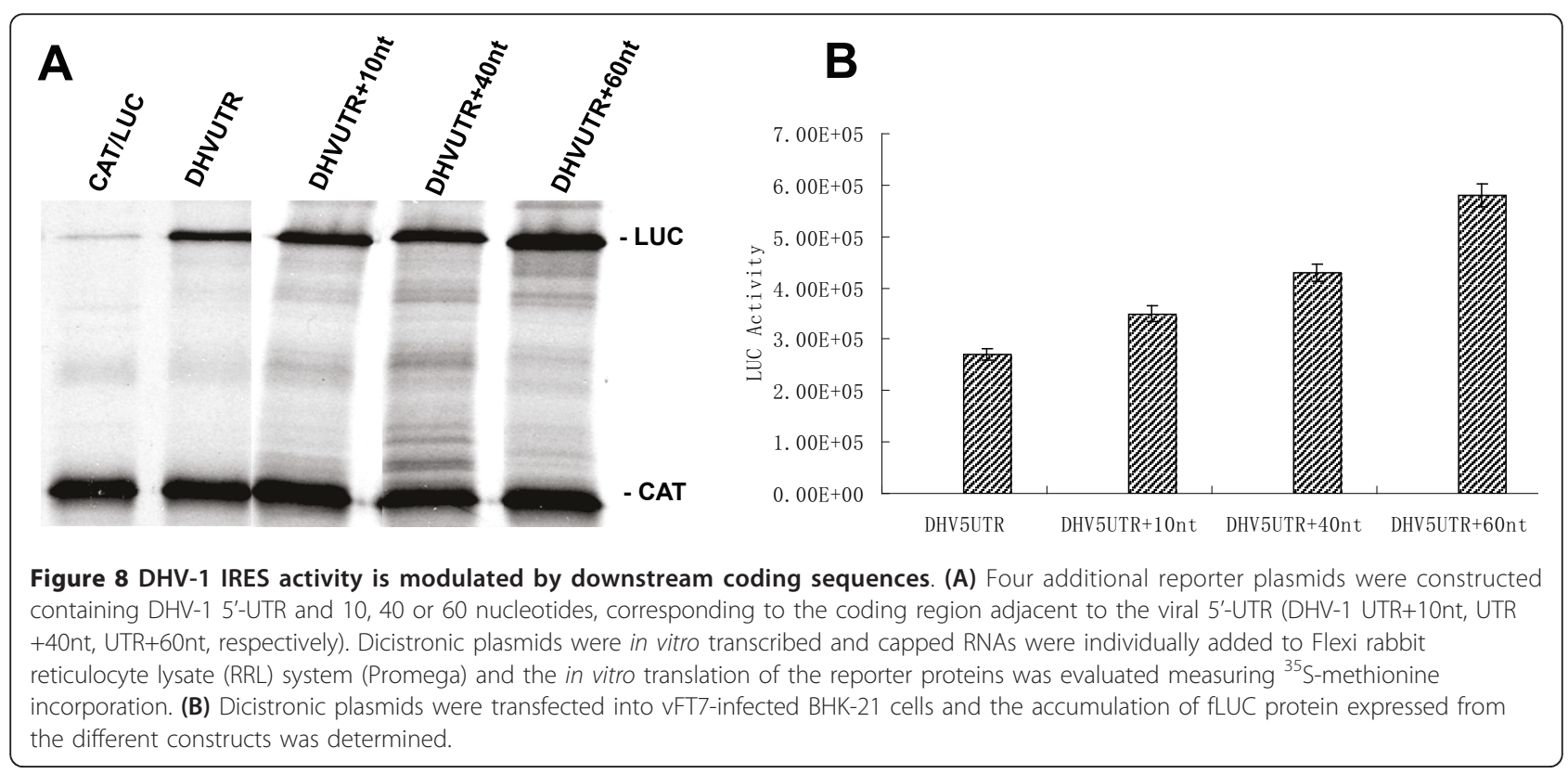


function. Moreover, the restoration of these structures partially recovered the activity of the DHV-1 IRES. These data provide strong evidence for the presence of the conserved domains II and III, common to other picornaviruses, within the DHV-1 5'-UTR and for the relevance of these regions to keep the normal internal initiation of translation of the DHV-1.

We have demonstrated that the sequences included within the DHV-1 Vp0 coding region contribute to the efficiency of internal initiation. The relevance of the coding sequences located immediately downstream of the IRESes has been previously described for other RNA viruses, such as the HCV or CSFV $[22,24]$. While most IRESs require only the 5'-non-translated region (5'-NTR) for full activity, the HCV or CSFV IRESes depend on the presence of protein-coding sequences downstream of the initiating AUG [37-39]. The minimal coding region elements required for high activity were exchanged between $\mathrm{HCV}$ and CSFV and the heterologous combinations were sufficiently active to rule out a highly specific functional interplay between the IRESes and the coding sequences [40]. Although nucleotide sequence of this region is different in CSFV and in $\mathrm{HCV}$, they share common features such as being A rich and not highly structured. In fact, the efficiency of internal initiation of these chimeric constructs correlated with the degree of single-strandedness of the region around the initiation codon. According to secondary structure prediction, the DHV-1 coding region located immediately downstream of the IRES is not highly structured. This sensitivity to secondary structure around the initiation codon could be due to the fact that the eIF4F complex (which includes the eIF4A helicase as one of its subunits) is not required for translation initiation on these IRESs. However, the interaction of cellular proteins with the coding regions adjacent to the IRESes has been proposed as an alternative mechanisms that enhance viral translation, as it is the case of the NS1associated protein 1 (NSAP1) and the HCV IRES [41], and cannot be discarded in the case of DHV-1.

Finally, we have characterized the DHV-1 IRES requirement for the eIF4F complex components and, as well as the EMCV and HCV-like IRESes, is insensitive to the FMDV L protease and the 4E-BP1 activities. These results indicate that the translation initiation takes places in the absence of fully functional eIF4E and eIF4G proteins. However, while the DHV-1 and HCVlike IRESes are not inhibited by hippuristanol or dominant negative forms of eIF4A, the EMCV IRES is strictly dependent on the eIF4A function [25]. Although the EMCV and DHV-1 IRESes share some common structural organization, they initiate translation by different mechanisms involving different cellular translation initiation factors. Moreover, as the DHV-1 and HCV share common structural motifs, they have similar requirement for the eIF4F complex components. Thus, it is likely that the translation initiation of DHV-1 could proceed similarly to PTV-1 and to other HCV-like IRES containing picornaviruses, by the direct recruitment of the $40 \mathrm{~S}$ ribosomal subunit to the viral mRNA.

All together, these results demonstrate, for the first time, the existence of an IRES element within the highly structured 5'-UTR region of the DHV-1 genome. The DHV-1 IRES could be classified as type IV IRES attending to its secondary structure and biological functions and, consequently, it exhibits functional similarities with the HCV IRES. These new advances in the understanding of the DHV-1 IRES structure, function and interaction with translation-initiation factors provides a foundation for developing therapeutics to prevent the viral protein synthesis.

\section{Materials and methods}

\section{RNA secondary structure prediction}

5'-UTR sequence of DHV-1 ZJ strain (GenBank No. EF382778) was aligned with other DHV-1 isolates from GenBank using DNAstar (DNASTAR Inc.) software. Secondary structure elements were predicted in Mfold [42].

\section{Plasmid construction}

DNA preparations were performed using standard methods as described in manufacturers' instructions. The reporter plasmids pGEM-CAT/EMC/LUC, containing the EMCV IRES CDNA, and pGEM-CAT/LUC were kindly offered by Ian Goodfellow (Imperial College, London, UK). These plasmids allow T7 promoter-directed expression of dicistronic mRNAs encoding chloramphenicol acetyl transferase (CAT) and firefly luciferase (LUC). For the construction of the DHV-1 5'-UTR-containing plasmid, a pair of primers (DHV-1 UTRF: 5'-GAGGATCCTTTGAAAGCGGGTGCATG-3'; DHV-1 UTRR: 5'-CGGGATTCTGCATGAAAG TCTACTGGT3') were used to amplify the DHV-1 5'-UTR from wild DHV-1 strain (ZJ-V, GenBank No. EF382778). The purified PCR fragments were digested with BamHI, and then inserted into similarly digested and phosphatased pGEMCAT/LUC between the two open reading frames (ORFs). The plasmid, containing the DHV-1 5'-UTR cDNA, was designated pGEM-CAT/DHV-1/LUC (Figure 1A). The further constructs containing the cDNA corresponding to the DHV-1 5'-UTR plus 10, 40, 60 nt of coding sequence were generated similarly using three pairs of specific primers and the resulting plasmids were termed pGEMDHVUTR+10nt, pGEM-DHVUTR+40nt, pGEMDHVUTR+60nt, respectively. Initiating AUG codon of the DHV-1 IRES was mutated to allow the expression of the luciferase from its own AUG. 


\section{Mutagenesis of the DHV-1 cDNA}

In order to introduce mutations in the predicted IIIe region, Quick-Change site-direct mutagenesis kit (Stratagene) was used in order to change the sequence in the loop region 570-573 from GAUA to AAAA. The plasmid pGEM-CAT/DHV-1UTR+60/LUC was used as the template with two specific primers: P1 (sense primer): 5'-CCTACACTGCCTAAAAGGGTCGCGGCTGGT-3'; P2 (antisense primer): 5' - CAGCCGCGACCCTTTTAGGCAGTGTAGGTT-3'. The resultant plasmid was named pGEMDHV IIIe mut. Similar strategies were used to introduce mutations within the stem sequences of the predicted pseudo-knots (termed Stem1-mut and Stem2mut). For the generation of the Stem1-mutant, the nts 447-449 (TGT) were changed to CCC. The mutagenic primers were as follows: P3 (sense primer): 5'-TGTAGGTGAGTGCCCGGTCTAGAGTAGGC-3'; P4 (anti-sense primer): 5'-CTACTCTAGACCGGGCACTCACCTACAAC-3'. For the generation of the Stem2 mutant, the nts 604-605 (CC) were changed to GG. The mutagenic primers were as follows: P5 (sense primer): 5'-TGATAGGGTCGCCCCTGGTCGAGTCC CA-3'; P6 (antisense primer): 5'-GGACTCGACC AGGGGCGACCCTATCAGG-3'. The presence of all the expected mutations in the plasmids (pGEMDHV IIIe mut, pGEMDHV S1 mut and pGEMDHV S2 mut) was confirmed by DNA sequencing. Similar strategies were used to introduce the compensatory mutations in Stem1 mut' and Stem2 mut' constructs.

\section{In vitro translation reactions}

For in vitro translation reactions, transcription of capped dicistronic mRNA was performed from the above plasmids, linearized with Xhol, using the Megascript transcription system (Ambion). Addition of the 7-mGTP cap 0 structure was performed using ScriptCap ${ }^{\mathrm{TM}} \mathrm{m}^{7} \mathrm{G}$ Capping System (Epicentre Biotechnologies) and mRNA was poly-adenylated using poly-A polymerase (PAP) following the suppliers' recommendations. In vitro transcribed mRNAs were added to the Flexi rabbit reticulocyte lysate (RRL) system (Promega) to a final concentration of $10 \mu \mathrm{g} / \mathrm{ml}$. This concentration of RNA was previously determined to give a linear yield of the translated product over the time course of the experiment $(90 \mathrm{~min})$. In reactions that required the addition of either recombinant $4 \mathrm{E}-\mathrm{BP} 1$, L-protease, the dominant negative mutant forms of eIF4A or hippuristanol, the RRL were pre-incubated with the recombinant proteins or with the eIF4A inhibitor at $30^{\circ} \mathrm{C}$ for 15 min prior to the addition of the different plasmids. After 90 min, the reactions were terminated by the addition of SDS-PAGE sample buffer and subsequently resolved on $12.5 \%$ polyacrylamide gels.

\section{Transient expression assays}

The dicistronic reporter plasmids $(1 \mu \mathrm{g})$ described above were transfected into BHK21 or DF-1 cells. Briefly, the plasmids were transfected into cells ( $35 \mathrm{~mm}$ dishes) previously infected with the recombinant vaccinia virus vTF7-3, which expresses T7 RNA polymerase, using Lipofectamine 2000 (Invitrogen) and Optimem (Gibco BRL). Cell lysates were prepared $24 \mathrm{~h}$ post-transfection and analyzed by SDS-PAGE and immunoblotting to determine CAT and LUC expression. Detection was achieved with anti-CAT (Sigma), anti-fLUC (Promega), peroxidase-labelled anti-rabbit (Amersham) or anti-goat (Dako Cytomation) antibodies respectively, using chemiluminescence reagents (Pierce). fLUC expression was also quantified using a firefly luciferase assay kit (Promega) with a luminometer according to the instructions of manufacturer.

\section{Recombinant proteins and reagents}

Recombinant His-4E-BP1 was generously supplied by I. Goodfellow (Imperial College London). L-protease was kindly provided by T. Skern (University of Viena). Dominant negative eIF4A mutants were generously supplied by I. Goodfellow (Imperial College London). Hippuristanol was kindly provided by J. Pelletier (McGill University).

\section{Acknowledgements}

We would like to thank Dr. lan G. Goodfellow, Department of Virology, Faculty of Medicine, Imperial College London, UK, for its great contribution to the design of the experiments and the set up of experimental conditions. This study was funded by grants from the Chinese Natural Sciences Foundation (30870114), National Key Laboratory of Veterinary Biotechnology (SKLVBF201004), the Special Fund for Agro-scientific Research in the Public Interest (No.201003012), the National advanced Technology Research and Development Program of China (863 Program) (No. 2011AA10A200).

\section{Author details}

${ }^{1}$ Shanghai Veterinary Research Institute, Chinese Academy of Agricultural Sciences, Shanghai, 200241, PR China. ${ }^{2}$ National Key Laboratory of Veterinary Biotechnology, Herbin, 150001, PR China. ${ }^{3}$ Centro Nacional de Biotecnología, CSIC Darwin 3, Cantoblanco 28049, Madrid, Spain.

\section{Authors' contributions}

$G L$ and EY carried out the design of the study, performed analyses of data and edited the manuscript. $\mathrm{CL}$ carried out the plasmid construction and mutagenesis of the DHV-1 CDNA. ZC conducted some experiments (For example, transient expression assays) in the study. All authors read and approved the final manuscript.

\section{Competing interests}

The authors declare that they have no competing interests.

Received: 22 December 2010 Accepted: 31 March 2011

Published: 31 March 2011

\section{References}

1. Levine PP, Fabricant J: A hitherto-undescribed virus disease of ducks in North. America Cornell Vet 1950, 40:71-86.

2. Kim MC, Kwon YK, Joh SJ, Lindberg M, Kwon JH, Kime JH, Kim SJ: Molecular analysis of duck hepatitis virus type 1 reveals a novel lineage close to the genus Parechovirus in the family Picornaviridae. J Gen Virol 2006, 87:3307-3316. 
3. Tseng CH, Knowles NJ, Tsai HJ: Molecular analysis of duck hepatitis virus type 1 indicates that it should be assigned to a new genus. Virus Res 2007, 123:190-203.

4. Racaniello VR: In Picornaviridae: the viruses and their replication. Fields Virology. Volume 1. 5 edition. Edited by: Knipe DM, Howley PM, Griffin DE. Lippincott Williams 2006:795-838.

5. Doudna J, Sarnow P: Translation initiation by viral internal ribosome entry sites. In Translational control in biology and medicine. Cold Spring Harbor monograph 48. Edited by: Mathews MB, Sonenberg N, Hershey JWB. Cold Spring Harbor Laboratory Press, Cold Spring Harbor, NY; 2007:129-153.

6. Gingras AC, Raught B, Sonenberg N: elF4 initiation factors: effectors of mRNA recruitment to ribosomes and regulators of translation. Annu Rev Biochem 1999, 68:913-963.

7. Kapp LD, Lorsch JR: The molecular mechanics of eukaryotic translation. Annu Rev Biochem 2004, 73:657-704.

8. Kieft JS, Zhou K, Jubin R, Murray MG, Lau JY, Doudna JA: The hepatitis C virus internal ribosome entry site adopts an ion-dependent tertiary fold. J Mol Biol 1999, 292:513-529.

9. Gray NK, Wickens M: Control of translation initiation in animals. Annu Rev Cell Dev Biol 1998, 14:399-458.

10. Hershey JWB: Translational control in mammalian cells. Annu Rev Biochem 1991, 60:717-755.

11. Pestova TV, Kolupaeva VG, Lomakin IB, Pilipenko EV, Shatsky IN, Agol IV, Hellen CU: Molecular mechanisms of translation initiation in eukaryotes. Proc Natl Acad Sci USA 2001, 98:7029-7036.

12. Belsham GJ, Jackson RJ: Translation initiation on picornavirus RNA. In 'Translational Control of Gene Expression' Monograph 39. Edited by: Sonenberg N, Hershey JWB, Mathews MB. Cold Spring Harbor Laboratory Press, Cold Spring Harbor, NY; 2000:869-900.

13. Bushell M, Sarnow P: Hijacking the translation apparatus by RNA viruses. J Cell Biol 2002, 158:395-399.

14. Kolupaeva VG, Pestova TV, Hellen CU, Shatsky IN: Translation eukaryotic initiation factor $4 \mathrm{G}$ recognizes a specific structural element within the internal ribosome entry site of encephalomyocarditis virus RNA. J Biol Chem 1998, 273:18599-18604.

15. Deniz N, Lenarcic EM, Landry DM, Thompson SR: Translation initiation factors are not required for Dicistroviridae IRES function in vivo. RNA 2009, 15:932-946.

16. Fraser CS, Doudna JA: Structural and mechanistic insights into hepatitis $C$ viral translation initiation. Nat Rev Microbiol 2007, 5:29-38.

17. Lancaster AM, Jan E, Sarnow P: Initiation factor-independent translation mediated by the hepatitis C virus internal ribosome entry site. RNA 2006, 12:894-902.

18. Pisarev AV, Shirokikh NE, Hellen CUT: Translation initiation by factor independent binding of eukaryotic ribosomes to internal ribosomal entry sites. C R Biol 2005, 328:589-605.

19. Belsham GJ: Divergent picornavirus IRES elements. Virus Res 2009, 139:183-192.

20. Dorner AJ, Semler BL, Jackson RJ, Hanecak R, Duprey E, Wimmer E: In vitro translation of poliovirus RNA: utilization of internal initiation sites in reticulocyte lysate. J Virol 1984, 50:507-514.

21. Ali IK, McKendrick L, Morley SJ, Jackson RJ: Activity of the hepatitis A virus IRES requires association between the cap-binding translation initiation factor (elF4E) and elF4G. J Virol 2001, 75:7854-7863.

22. Honda M, Beard MR, Ping LH, Lemon SM: A phylogenetically conserved stem-loop structure at the $5^{\prime}$ border of the internal ribosome entry site of hepatitis $C$ virus is required for cap-independent viral translation. J Virol 1999, 73:1165-1174.

23. Kaku Y, Chard LS, Inoue T, Belsham GJ: Unique characteristics of a picornavirus internal ribosome entry site from the porcine teschovirus-1 Talfan. J Virol 2002, 76:11721-11728.

24. Rijnbrand RC, Abbink TE, Haasnoot PC, Spaan WJ, Bredenbeek PJ: The influence of AUG codons in the hepatitis $C$ virus $5^{\prime}$ nontranslated region on translation and mapping of the translation initiation window. Virology 1996, 226:47-56

25. Chard LS, Bordeleau ME, Pelletier J, Tanaka J, Belsham GJ: Hepatitis C virusrelated internal ribosome entry sites are found in multiple genera of the family Picornaviridae. J Gen Virol 2006, 87:927-936.

26. Bakhshesh M, Groppelli E, Willcocks MM, Royall E, Belsham GJ, Roberts LO: The picornavirus avian encephalomyelitis virus possesses a hepatitis $C$ virus-like internal ribosome entry site (IRES) element. J Virol 2008, 82:1993-2003.

27. Hellen CU, de Breyne S: A distinct group of hepacivirus/pestivirus-like internal ribosomal entry sites in members of diverse picornavirus genera: evidence formodular exchange of functional noncoding RNA elements by recombination. J Virol 2007, 81:5850-5863.

28. Lukavsky PJ, Otto GA, Lancaster AM, Sarnow P, Puglisi JD: Structures of two RNA domains essential for hepatitis $C$ virus internal ribosome entry site function. Nat Struct Biol 2000, 7:1105-1110.

29. Brown EA, Zhang H, Ping LH, Lemon SM: Secondary structure of the 5'nontranslated regions of hepatitis $C$ virus and pestivirus genomic RNAs. Nucleic Acids Res 1992, 20:5041-5045.

30. Gingras AC, Gygi SP, Raught B, Polakiewicz RD, Abraham RT, Hoekstra MF, Aebersold R, Sonenberg N: Regulation of 4EBP1 phosphorylation: a novel two-step mechanism. Genes Dev 1999, 13:1422-1437.

31. Svitkin YV, Herdy B, Costa-Mattioli M, Gingras AC, Raught B, Sonenberg N: Eukaryotic translation initiation factor $4 \mathrm{E}$ availability controls the switch between cap-dependent and internal ribosomal entry site-mediated translation. Mol Cell Biol 2005, 25:10556-10565.

32. Pause $A$, Méthot $N$, Sonenberg N: The HRIGRXXR region of the DEAD box RNA helicase eukaryotic translation initiation factor $4 A$ is required for RNA binding and ATP hydrolysis. Mol Cell Biol 1993, 13:6789-98.

33. Svitkin $\mathrm{V}$, Pause A, Haghighat A, Pyronnet S, Witherell G, Belsham GJ, Sonenberg N: The requirement for eukaryotic initiation factor 4A (elF4A) in translation is in direct proportion to the degree of mRNA $5^{\prime}$ secondary structure. RNA 2001, 7:382-394

34. Bordeleau M, Mori A, Oberer M, Lindqvist L, Chard LS, Higa T, Belsham GJ, Wagner G, Tanaka J, Pelletier J: Functional characterization of IRESes by an inhibitor of the RNA helicase elF4A. Nat Chem Biol 2006, 2:213-220

35. Brown EA, Ehrenfeld E: Translation of poliovirus RNA in vitro: changes in cleavage pattern and initiation sites by ribosomal salt wash. Virology 1979, 97:396-405.

36. Pisarev AV, Chard LS, Kaku Y, Johns HL, Shatsky IN, Belsham GJ: Functional and structural similarities between the internal ribosome entry sites of hepatitis C virus and porcine teschovirus, a picornavirus. J Virol 2004, 78:4487-4497.

37. $\mathrm{Lu} \mathrm{HH}$, Wimmer E: Poliovirus chimeras replicating under the translational control of genetic elements of hepatitis $C$ virus reveal unusual properties of the internal ribosomal entry site of hepatitis $C$ virus. PNAS 2006, 93:1412-1417.

38. Reynolds JE, Kaminski A, Kettinen HJ, Grace K, Clarke BE, Carroll AR, Rowlands DJ, Jackson RJ: Unique features of internal initiation of hepatitis C virus RNA translation. EMBO J 1995, 14:6010-20.

39. Fletcher SP, Ali IK, Kaminski A, Digard P, Jackson RJ: The influence of viral coding sequences on pestivirus IRES activity reveals further parallels with translation initiation in prokaryotes. RNA 2002, 8:1558-71.

40. Fletcher SP, Jackson RJ: Pestivirus internal ribosome entry site (IRES) structure and function: elements in the $5^{\prime}$ untranslated region important for IRES function. J Virol 2002, 76:5024-5033.

41. Kim JH, Paek KY, Ha SH, Cho S, Choi K, Kim CS, Ryu SH, Jang SK: A cellular RNA-binding protein enhances internal ribosomal entry site-dependent translation through an interaction downstream of the hepatitis $C$ virus polyprotein initiation codon. Mol Cell Biol 2004, 24:7878-7890.

42. Zuker M: Mfold web server for nucleic acid folding and hybridization prediction. Nucleic Acids Res 2003, 31:3406-3415.

doi:10.1186/1743-422X-8-147

Cite this article as: Liu et al: The duck hepatitis virus $5^{\prime}$-UTR possesses HCV-like IRES activity that is independent of elF4F complex and modulated by downstream coding sequences. Virology Journal 2011 8:147. 